\title{
CONF-960569--21
}

GA-A22358

\section{ARRAY OF NEUTRAL PARTICLE ANALYZERS AT DIII-D}

\author{
by \\ E.M. CAROLIPIO and W.W. HEIDBRINK
}

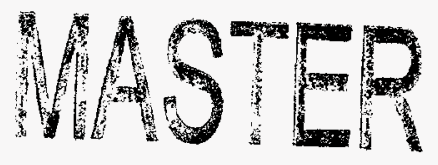

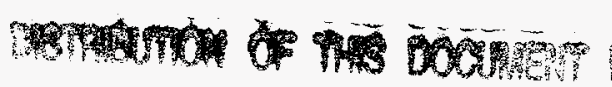

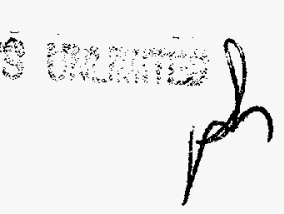

DECEMBER 1996 


\section{DISCLAIMER}

This report was prepared as an account of work sponsored by an agency of the United States Government. Neither the United States Government nor any agency thereof, nor any of their employees, makes any warranty, express or implied, or assumes any legal liability or responsibility for the accuracy, completeness, or usefulness of any information, apparatus, product, or process disclosed, or represents that its use would not infringe privately owned rights. Reference herein to any specific commercial product, process, or service by trade name, trademark, manufacturer, or otherwise, does not necessarily constitute or imply its endorsement, recommendation, or favoring by the United States Government or any agency thereof. The views and opinions of authors expressed herein do not necessarily state or reflect those of the United States Government or any agency thereof. 


\section{DISCLAMMIER}

Portions of this document may be illegible in electronic image products. Images are produced from the best available original document. 


\title{
ARRAY OF NEUTRAL PARTICLE ANALYZERS AT DIII-D
}

RECEIVED

by E.M. CAROLIPIO ${ }^{\dagger}$ and W.W. HEIDBRINK $\dagger$

This is a preprint of a paper to be presented at the 12th International Conference on Plasma Surface Interactions in Controlled Fusion Devices, May 20-24, 1996, Saint-Raphael, France and to be published in the Proceedings.

†University of California, Irvine

\author{
Work supported by \\ the U.S. Department of Energy \\ under Contract No. DE-AC03-89ER51114 \\ and Subcontract No. SC-L134501
}

GA PROJECT 3466

DECEMBER 1996 


\section{ABSTRACT}

Local measurements of the fast-ion distribution in auxiliary-heated plasmas are key to understanding the behavior of energetic particles under a variety of conditions, such as beam-ion transport during Alfvén instabilities and the acceleration of beam ions by fast waves. For the first time at DIII-D, lineaveraged and local measurements of the energetic-particle density (for $E=5-75 \mathrm{keV}$ ) are possible using an array of four compact charge-exchange analyzers. ${ }^{1}$ The installation consists of three vertically-viewing analyzers with fixed sightlines, measuring particles with $\chi=90^{\circ}$ (where $\chi$ is the angle between the particle's velocity and the toroidal direction) and one horizontally-viewing analyzer with a variable sightline, measuring particles with $2^{\circ} \mathrm{U} \chi \grave{\mathrm{U}} 60^{\circ}$. All the analyzers can make passive measurements while three detectors, with sightlines that intersect deuterium heating beams, can make active charge-exchange measurements.

${ }^{1}$ P. Beiersdorfer et al., Rev. Sci. Instrum. 58, 2092 (1987). 


\section{INTRODUCTION}

Diagnosing the fast-ion population is important in auxiliary-heated plasmas. Ideally, measurements would give details of the fast-ion distribution function $f_{b}$ in space $\vec{r}$, energy $E$, and pitch-angle $\chi$. (Here $\chi$ is the angle which the particle's velocity $\vec{v}$ makes with respect to the unit vector in the toroidal direction $\hat{e}_{\phi}$, i.e. $\cos \chi=v_{\varphi \beth} J v$.) At DIII-D, data from diagnostics that measure the $2.5 \mathrm{MeV}$ neutron production rate [1] coupled with the lineaveraged electron density yield the total number of beam ions [2], but the diagnostics effectively average over $\vec{r}, E$, and $\chi$. Understanding the local behavior of fast ions is critical for several experiments, such as beam-ion loss during Alfvén instabilities [2] and the acceleration of beam ions by fast-waves [3]. Consequently, local measurements of the distribution function are desirable. Charge-exchange analyzers are able to measure a single point $f_{b}(\vec{r}, E, \chi)$ in the distribution function. The depth into the plasma from which ions can be detected by charge-exchange analyzers is inversely proportional to the line density; given the modest sizes $(R \sim 1.67 \mathrm{~m}, a \grave{\mathrm{U}} 67 \mathrm{~cm})$ and densities $\left(n_{e} \sim 10^{19} \mathrm{~m}^{-3}\right)$ of DIII-D plasmas, these analyzers can detect neutrals coming from the core.

A charge exchange analyzer had been installed on the DIII-D tokamak but could never take data during neutral-beam injection. The "E-parallel-to-B" (E_B) charge-exchange analyzer[4] used a He stripping cell to reionize the neutrals and electric and magnetic fields to deflect the particles onto a microchannel detecting plate [5]. The microchannel plate was far too sensitive to neutrons: even with $\sim 4 \mathrm{~cm}$ of lead shielding, the output signal was saturated by a $2.5 \mathrm{MeV}$ neutron flux of $\sim 10^{12} \mathrm{n} / \mathrm{s} \cdot \mathrm{m}^{2}$, roughly the flux at the radial location of the plate produced by the injection of a single deuterium beam into a deuterium target plasma. A charge-exchange analyzer $[6,7]$ using a channel electron multiplier, or "channeltron", to detect reionized particles is a more effective charge-exchange diagnostic for DIII-D. The channeltron is much less sensitive to neutrons than the microchannel plate and operates at fluxes in excess of $10^{14} \mathrm{n} / \mathrm{s} \cdot \mathrm{m}^{2}$ (corresponding to neutron rates of over $10^{16} \mathrm{n} / \mathrm{s}$ ) with no auxiliary neutron shielding.

This paper describes installation of four compact, energy-resolving analyzers on the DIII-D tokamak. The analyzers [6], originally built and operated at the Princeton Plasma Physics Laboratory, come equipped with channeltrons and are capable of detecting neutrals with energies between 5 and $75 \mathrm{keV}$. Since nearly all DIII-D discharges are beam-heated D-D plasmas, mass resolution is not a mandatory feature for an analyzer on this tokamak. The 
array gives four line-averaged and three local measurements of the beam-ion density for a spectrum of energies and pitch-angles.

The horizontal analyzer is described in Section I; the other three have similar characteristics. In Section II, the layout of the array, with a single, horizontally-scanning analyzer at the midplane and three fixed, vertically-viewing analyzers, is discussed. Data from the horizontal analyzer are presented in Section III. 


\section{DESCRIPTION OF INSTRUMENT}

All four analyzers are similar in construction and simple in design (Fig. 1). The incoming particle flux is ionized by a $100 \AA$-thick carbon foil [8] mounted on a $90 \%$ transmission nickel mesh. The mesh is attached to an aluminum disk $1.0 \mathrm{~cm}$ in diameter with an aperture of $0.5 \mathrm{~cm}$. Two cylindrically-shaped parallel plates, energized to a potential $V_{\text {plate }}$, deflect the reionized particles $90^{\circ}$ to a channeltron detector [5] running in analog (current) mode. A preamplifier acts as a current-to-voltage converter and transmits the signal to a digitizer.

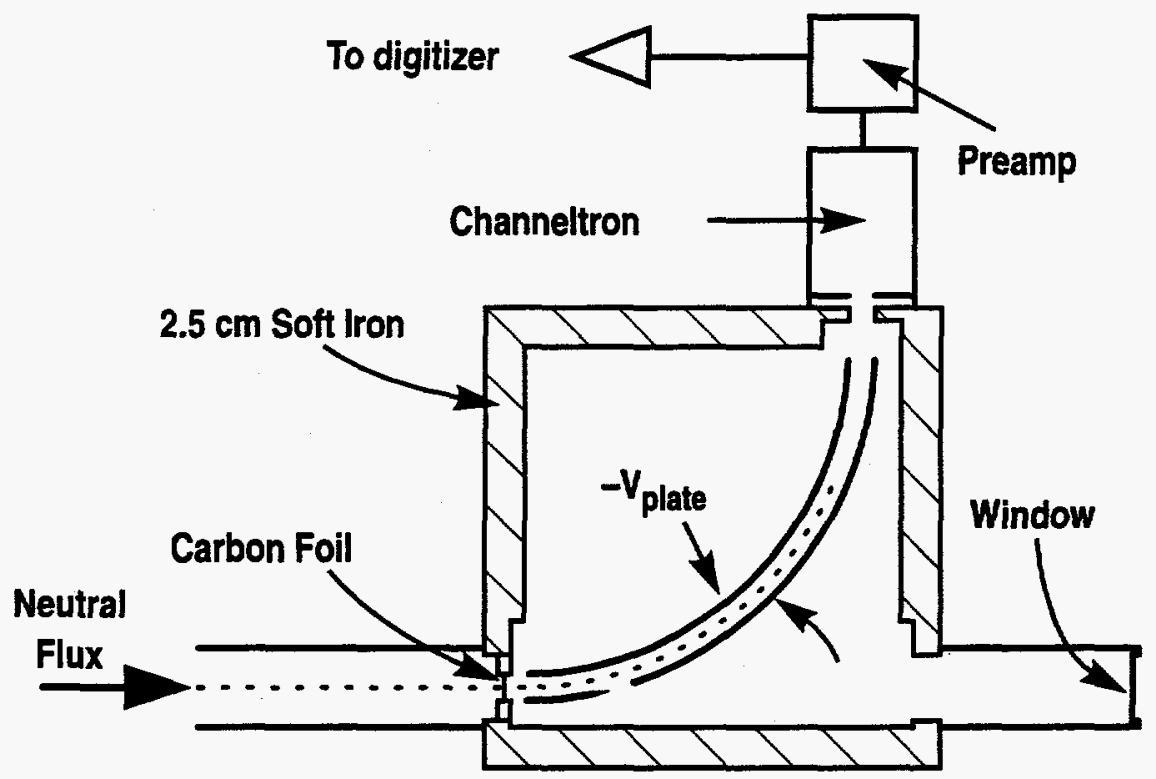

Fig. 1. Schematic of a compact charge-exchange analyzer (not to scale). The analyzer has external dimensions of $18 \times 18 \times 13 \mathrm{~cm}$ with an outer housing made of $2.5 \mathrm{~cm}$ thick soft iron for magnetic shielding. The upper and lower plates have radii of 9.8 and $11.1 \mathrm{~cm}$ respectively. A through-hole is drilled in the bottom plate to allow for sightline alignment of the diagnostic. The mesh and foil are positioned $1.2 \mathrm{~cm}$ from the deflection plates by a fabricated stainless piece (not shown) press-fitted into the nipple on the front (machine-side) of the analyzer. The piece is designed so gas flows around the disk, preventing a pressure differential across the foil during pump-down. A window is used to visually inspect the foil while the system is under vacuum.

Two Trek high voltage power supplies [9] provide positive and negative potentials to the lower and upper electrostatic plates respectively. Although the Treks have a maximum output of $10 \mathrm{kV}$, an operational limit of $8.7 \mathrm{kV}$ is placed on the supplies to prevent arcing. A frequency generator controls the Trek output. The generator supplies either a DC control 
voltage to deflect particles of a specific energy or a triangle-wave control voltage ( $\left.\tau_{\text {sweep }} \grave{\mathrm{A}} 0.1-0.5 \mathrm{~s}\right)$ with a DC offset to deflect particles with a spectrum in energy ( $E_{\max }$ $\left.E_{\min } \mathrm{U} 75 \mathrm{keV}\right)$ at the expense of time resolution.

Beiersdorfer et al. [6] quantitatively studied the characteristics of this design. The analyzer was tested on a $100 \mathrm{keV}$ ion-beam test stand [10] at the DIII-D facility and the calibration was consistent with their results. For a monoenergetic ion beam, the particle energy $E_{0}$ as a function of plate voltage $V_{\text {plate }}$ with a foil placed along the beam trajectory was found to vary as (for $E_{0} \mathrm{U} 5 \mathrm{keV}$ )

$$
E_{0}(\mathrm{keV})=8.85 V_{\text {plate }}(\mathrm{kV})+1.75 .
$$

This relationship is true for both protons and deuterons. The energy lost by particles passing through a $100 \AA$-thick foil $\Delta E_{\text {foil }}$ was measured to be (for $E_{0} \mathrm{U} 5 \mathrm{keV}$ )

$$
\Delta E_{\text {foil }}(\mathrm{keV}) \approx 0.44 \sqrt{E_{0}(\mathrm{keV})} .
$$

The energy resolution of the analyzers $\delta_{\beth} E / E$ is about $0.048[6]$ since no baffles are placed along the sightline.

During tokamak operations, the channeltron also detects neutrons and gammas generated by $d(d, n)^{3} \mathrm{He}$ reactions, but this effect can be subtracted out. The amplitude and timeevolution of the neutron-induced signal is measured by closing the gate valve to the vessel. The resulting signal closely follows the $2.5 \mathrm{MeV}$ neutron production rate as measured by plastic scintillators cross-calibrated to an array of neutron counters [1]. This measurement typically yields a background signal (in volts) of $\sim 7 \% 10^{-17} S_{n}$, where $S_{n}$ is the neutron rate in $\mathrm{n} / \mathrm{s}$, assuming a normal bias $(2.4 \mathrm{kV})$ is applied to the channeltron. In most cases, this background signal is negligible. (For the discharge shown in Fig. 4, with $S_{n}=1.8 \% 10^{14} \mathrm{n} / \mathrm{s}$, the neutron-induced signal is $\sim 0.02 \mathrm{~V}$ and, for active charge exchange, the signal-to-noise ratio is much greater than unity.) For high performance discharges $\left(S_{n} \mathrm{U}^{1} 10^{16} \mathrm{n} / \mathrm{s}\right)$, the neutron noise is significant but never saturates the signal.

Plasma light is another potential source of noise. Though studied only qualitatively, the effect of stray light at the horizontal location was found to be negligible. For several shots, the plates were reverse-biased with the gate valve open. This ensures that the diagnostic views the plasma but no reionized neutrals are deflected by the channeltron. The signal correlated well with the $2.5 \mathrm{MeV}$ neutron production rate but had no correlation with the $D_{\alpha}$ or soft x-ray signals. 


\section{ARRAY LAYOUT}

One analyzer arrived at DIII-D in 1989. The analyzer was mounted directly on the innermost vertically-viewing port on top of the vessel (see Fig. 2) and operated for several months but never collected valid data. The problem was later traced to the stray magnetic field, produced primarily by the Ohmic heating coils, at the analyzer's location $(R=1.46 \mathrm{~m}$, $Z=2.99 \mathrm{~m}$ ). This field, which can be as large as $2.8 \mathrm{kG}$, is strong enough to saturate the soft iron shielding and affect the reionized neutral's trajectory inside the shield box. The same analyzer was later installed along the midplane (in place of the E_B analyzer) where the stray fields are an order of magnitude less than at the previous location; here, the analyzer successfully collected valid data.

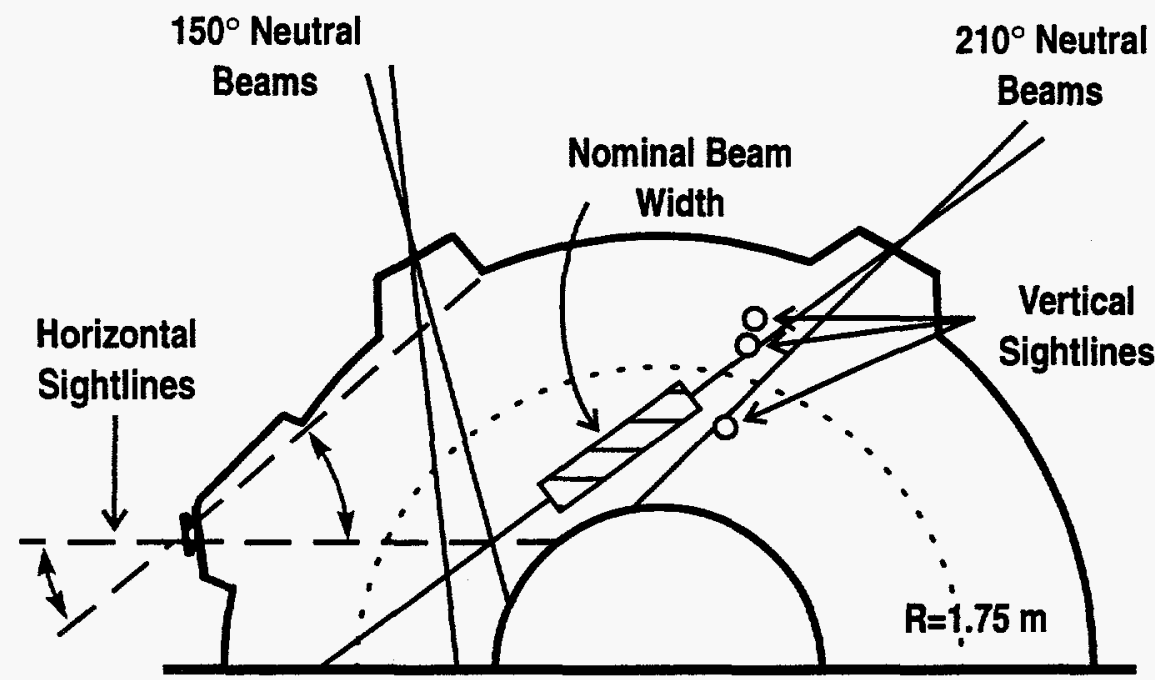

Fig. 2. Plan view of the DIII-D tokamak showing the $150^{\circ}$ and $210^{\circ}$ degree beam-lines (solid), the horizontal analyzer sightline (dash), and the vertical analyzer sightlines (open circles). For DIII-D, the nominal beam width is $20 \mathrm{~cm}$ and is indicated on the figure for the $210^{\circ}$ Left beam-line (box with cross-hatches). For clarity, the sightlines for the vertical analyzers have been exaggerated. Also shown (dot) is the magnetic axis of a DIII-D plasma with $R=1.75 \mathrm{~m}$. The horizontal analyzer can scan between the indicated sightlines. Three of the four analyzers can be used for active charge-exchange measurements: the horizontal analyzer with both $150^{\circ}$ beams, the innermost vertical analyzer with the $210^{\circ}$ Right beam, and the central vertical analyzer with the $210^{\circ}$ Left beam.

Four analyzers are currently installed on the DIII-D tokamak: their sightlines, and the centerlines for the $150^{\circ}$ and $210^{\circ}$ beams, are shown in Fig. 2 . Three analyzers are mounted with vertically-viewing sightlines. The one previously installed on the machine retains its horizontal sightline along the midplane. 
The strength of stray magnetic fields was a problem at the vertical location so, in the new installation, the vertical analyzers are mounted further away from the vessel at $Z=3.93 \mathrm{~m}$. The stray fields here $(1.2,0.98$, and $0.87 \mathrm{kG}$ from $R=1.46,1.94$, and $2.10 \mathrm{~m}$, respectively) are low enough that no additional magnetic shielding is necessary. All three are pumped by a single turbo/backing pump combination.

The horizontally-viewing analyzer, with its own pumping system, rides on a newlydesigned cart. The cart is attached to a pivot at the machine end and to a linear bearing mounted on a track at the far end. A welded bellows connects the diagnostic's vacuum assembly to the vessel port. The cart can pivot from $15^{\circ}$ to $60^{\circ}$ relative to the line perpendicular to the torus passing through the pivot point; a position transducer [11] measures its position.

At DIII-D, eight neutral beam sources are housed in four beam boxes; for each box, a "Left" and "Right" source inject neutral particles into the torus at tangency radii of $R_{\text {tan }}=1.10$ and $0.74 \mathrm{~m}$, respectively. For the horizontal analyzer, either source in the $150^{\circ}$ beam box can provide a local source of neutrals for active charge-exchange measurements. (Although the horizontal sightline intersects the $210^{\circ}$ beam-lines, these sources are ineffective for active measurements since the beam and the escaping neutral particle flux are too strongly attenuated to discern an appreciable signal.) The Left and Right $210^{\circ}$ sources can be used as doping beams for the innermost and central vertical analyzers respectively.

Since the horizontal analyzer's sightline is adjustable, it measures particles with various pitch angles $\chi$. In Fig. 3, $\chi$ for detected particles as a function of the major radius $R$ at which the analyzer sightline and the $150^{\circ}$ beam-lines intersect is shown. The horizontal analyzer can detect particles with $2^{\circ} \grave{\mathrm{U}} \chi \grave{\mathrm{U}} 60^{\circ}$ while all three vertical analyzers measure particles with $\chi=90^{\circ}$. Also shown are the pitch angles of injected neutrals from both $150^{\circ}$ beam orientations as a function of the major radius at which they ionize.

Both the neutral density $n_{0}$ and the beam-ion distribution function $f_{b}$ determine the strength of the active charge-exchange signal. The neutral density peaks for large values of $R$ since attenuation of the beam is minimized. On the other hand, for smaller values of $R, f_{b}$ tends to increase because the detected pitch angle is closer to the pitch angles of the injected beams. A third factor is the beam deposition: this is usually largest near the magnetic axis. For most applications, the horizontal analyzer is set such that the sightline intersects the Left beam at $R=1.84 \mathrm{~m}$ since this location is near the magnetic axis. 


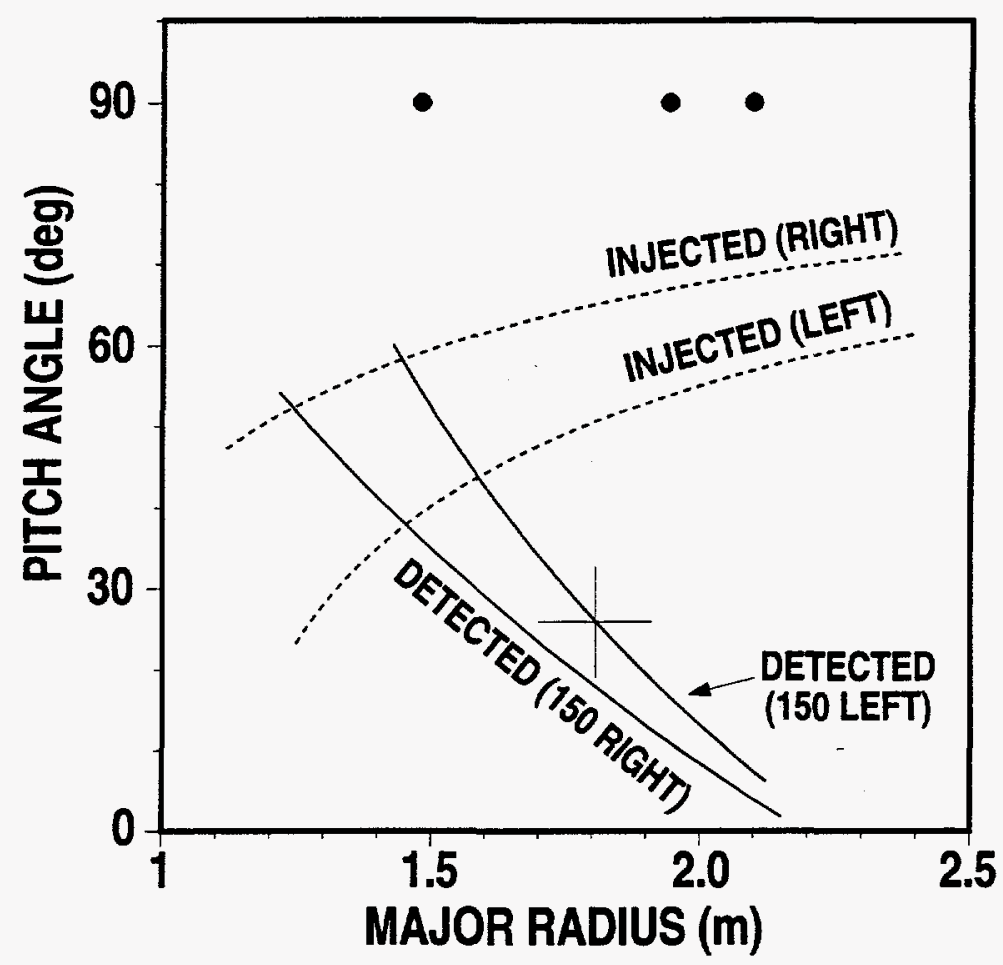

Fig. 3. Pitch angle $\chi$, where $\cos \chi=v_{\phi \supseteq} / v$, versus major radius $R$. The dashed lines show the pitch angles of ions born near $R$. The solid lines show the pitch angles accessible to the horizontal analyzer when using the $150^{\circ}$ beams for active charge-exchange measurements; the error bar shows the total spread in major radius and pitch angle associated with the beam footprint. (The 1/e intensity points are approximately half as large.) The sightlines of the vertical analyzers (circles) are also indicated.

The resolution of the active charge-exchange measurements is determined primarily by the spatial extent of the neutral source provided by the heating beams. For the horizontal measurements, the resolution in major radius is typically $\sim 20 \mathrm{~cm}$ (Fig. 3). In contrast, the vertical measurements are well resolved in pitch angle and major radius, but span $\sim 40 \mathrm{~cm}$ in the vertical direction. 


\section{TOKAMAK RESULTS}

Sample data from the horizontal analyzer are shown in Fig. 4(a). Three different sources are employed for this discharge but the beams are programmed so only one source at a time is in use. The analyzer is set to detect $\sim 50 \mathrm{keV}$ particles. The signal more than doubles when neutrals from the $150^{\circ}$ neutral beam intersects the analyzer sightline. The background passive signal arises from collisions with edge neutrals. A transition from the L-mode confinement regime to the $\mathrm{H}$-mode occurs at $\sim 1.61 \mathrm{~s}$; this has a larger impact on the passive signal than on the active signal produced by the injected neutrals. To obtain the active signal [Fig. 4(b)], measurements of the beam timing are used to subtract the passive signal from the total signal. As the discharge evolves, the plasma density rises. This reduces the active signal for two reasons: the penetration of the injected $150^{\circ}$ neutrals is reduced and reionization losses of escaping neutrals are increased. In order to infer the beam-ion density, it is necessary to correct for each of these effects. Model profiles, atomic cross sections, and the measured line density are used to calculate the corrected data shown in Fig. 4(c). (Work is in progress to develop a computer code that uses the measured density and temperature profiles in a more accurate calculation of attenuation effects.) For this particular orientation of the analyzer, the signals from the Left and Right $150^{\circ}$ sources originate from nearly the same position in the plasma; with the attenuation correction, the agreement between the corrected signals from each source is excellent.

This local charge-exchange measurement of beam density is compared with the average beam density inferred from the neutron rate in Fig. 4(c). For the conditions of this discharge, beam-plasma reactions predominate, so the total beam density $N_{b}$ (averaged over position and fusion cross section) is proportional to the neutron rate $S_{n}$ divided by the number of target deuterons (which is proportional to the electron density for this DIII-D plasma with a low value of $Z_{\text {eff }}$. In this quiescent discharge, the time evolution of the two signals is similar.

As shown in Fig. 4(c), attenuation effects are modest in low-density DIII-D plasmas $\left(\overline{n_{e}} \mathrm{U} 3 \% 10^{19} \mathrm{~m}^{-3}\right)$. However, in high-density plasmas $\left(\overline{n_{e}} \mathrm{U} 8 \% 10^{19} \mathrm{~m}^{-3}\right)$, the line density is too large for active-charge exchange measurements. Also, even at moderate values of line density, active charge-exchange measurements from the inside of the torus (U்1.4 m) are problematic because of the relatively long path lengths. 

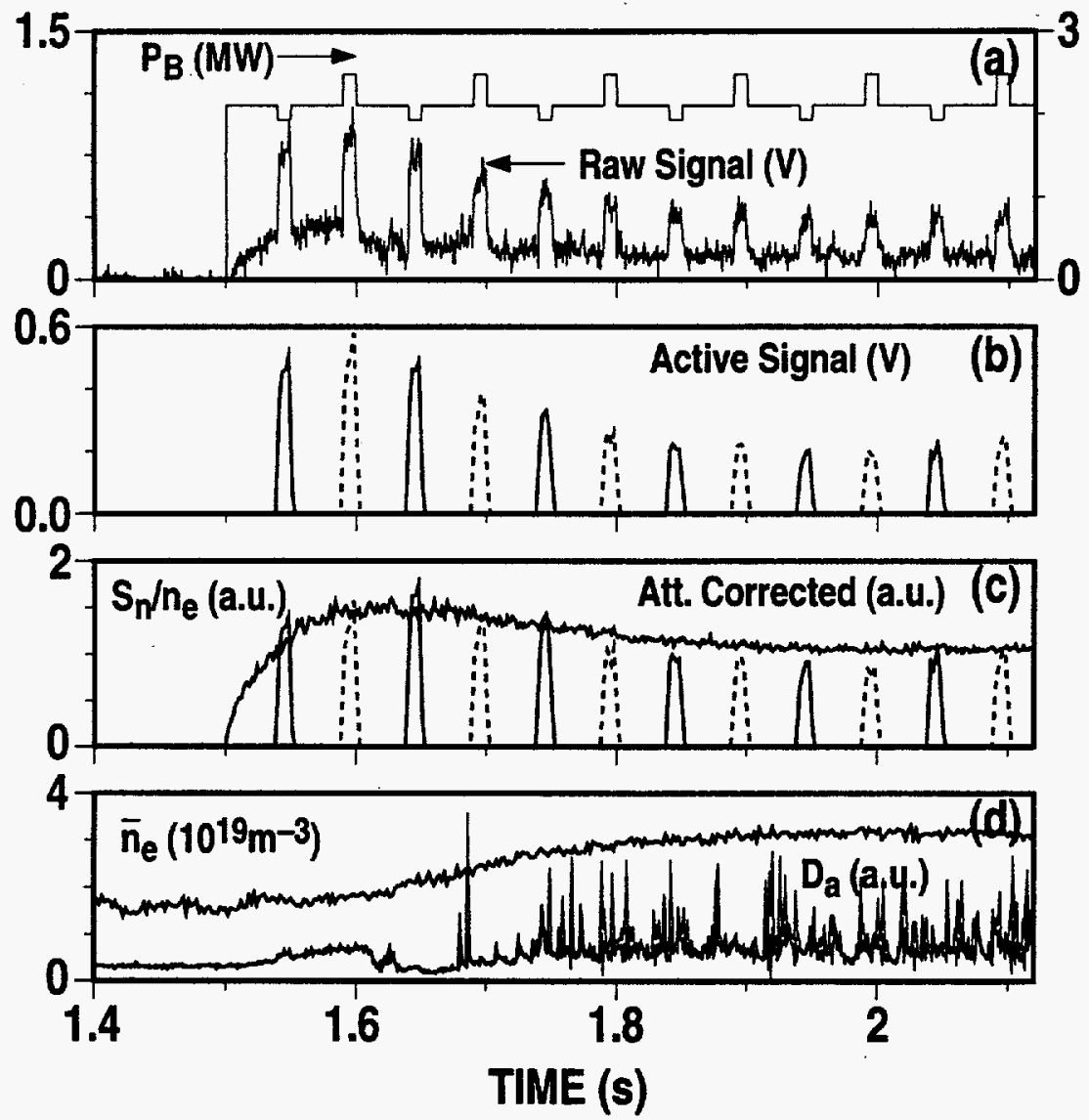

Fig. 4. (a) Time evolution of the injected beam power and the horizontal charge-exchange signal. Although only one neutral beam source injects at a time, three sources are employed: $330^{\circ}$ Left $(75 \mathrm{kV}, 2.1 \mathrm{MW}), 150^{\circ}$ Left $(63 \mathrm{kV}, 1.9 \mathrm{MW})$, and $150^{\circ}$ Right ( $75 \mathrm{kV}, 2.5 \mathrm{MW}$ ). Neutrals from the $150^{\circ}$ Left and Right beams intersect the analyzer sightline at major radii of 2.04 and $2.02 \mathrm{~m}$, respectively. (b) Active charge-exchange signal from the $150^{\circ}$ Left (solid) and $150^{\circ}$ Right (dash) beams obtained by subtracting the passive signal from the raw signal. (c) Active signals from the $150^{\circ}$ Left (solid) and $150^{\circ}$ Right (dash) beams including attenuation corrections. The calculations use the experimental values of $\overline{n_{e}}$ and $P_{b}$ with model profiles to calculate the attenuation of the neutral beams (modeled as "pencil" beams) and the reionization of the escaping neutrals. Also shown is the neutron rate $S_{n}$ divided by the line density $\bar{n}_{e}$, which is an empirical volume-averaged measurement of the beam-ion density. (d) Divertor $D_{\alpha}$ light and line average electron density from a horizontally-viewing interferometer chord. (Shot $\# 81401: I_{p}=0.6 \mathrm{MA} ; B_{T}=2.1 \mathrm{~T}$; double-null divertor configuration.) 


\section{REFERENCES}

[1] W.W. Heidbrink, P.L. Taylor, J.A. Phillips, "Measurements of the Neutron Source Strength at DIII-D", these proceedings.

[2] H.H. Duong et al., Nuclear Fusion 33, 749 (1993).

[3] S.C. Chiu et al., Proceedings of the 11th Topical Conference on Radio Frequency Power in Plasmas (1995).

[4] W.W. Heidbrink, Rev. Sci. Instrum. 59, 1681 (1988).

[5] Galileo Electro-Optics Co., Sturbridge, MA 01418.

[6] P. Beiersdorfer, A.L. Roquemore, and R. Kaita, Rev. Sci. Instrum. 58, 2092 (1987).

[7] S.L. Davis, D. Mueller, and C.J. Keane, Rev. Sci. Instrum. 54, 315 (1983).

[8] Lebow Company, 5960 Mandarin Ave., Goleta, CA 93117.

[9] Trek Inc., 3932 Salt Works Rd., Medina, NY 14103-0728.

[10] General Ionex Corp., 25 Hayward St., Ipswich, MA 01938.

[11] Houston Scientific, 4202 Director's Row, Houston, TX 77092. 



\section{ACKNOWLEDGMENT}

We wish to acknowledge and thank the following individuals for their assistance in completing this project: L. Roquemore for the analyzers; T. Carlsness, J. Evans. R. Ellis, R. Geer, T. Holoman, J.R. Robinson, G. Rolens, and R. Wood for their assistance with the hardware; R. Kaita for the calibration data; A. Molvik and C. Greenfield for contributions to Fig. 3; S. Scott for the TEXAS analysis code, and M. Brown, G. Bramson, and Yu Sun for subsequent modifications to the code; and C. Parker, R. Snider, and P.L. Taylor for their suggestions and advice. We are also grateful to the members of the DIII-D Team for their operational support during experiments.

This work was supported under the U.S. Department of Energy Contract No. DE-AC03-89ER51114, Subcontract SC-L134501. 